
The Dibner Library of the History of Science and Techology

SMITHSONIAN INSTITUTION LIBRARIES

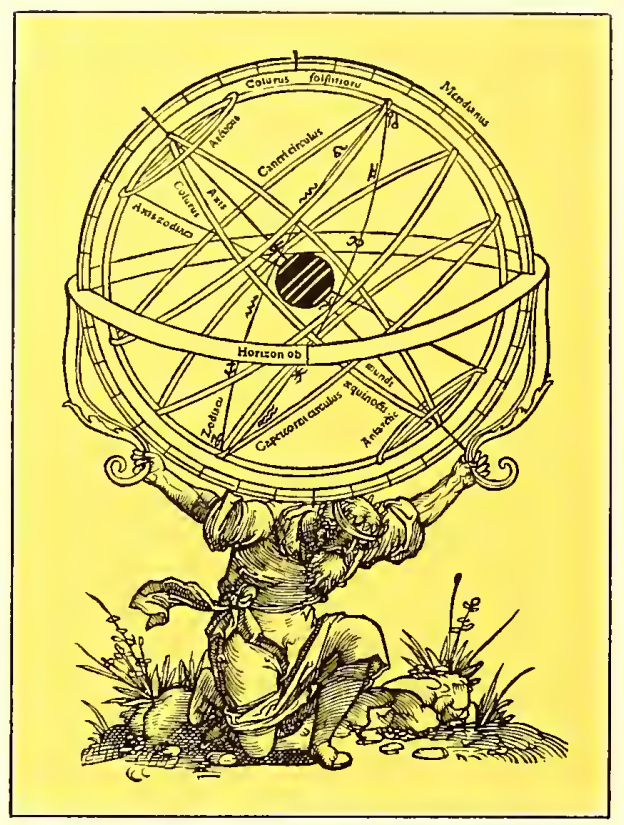










\section{AMENDED SPECIFICATION.}

Reprinted as amended in accordance with the decision of the Chief Examiner dated the 21st day of February 1899.

$\mathrm{N}^{\circ} 29,306$

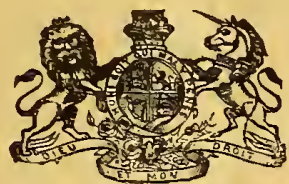

\section{A.D. 1897}

Date of Application, 10th Dec., 1897

Complete Specification Left, 12th Sept., 1898-Accepted, 15th 0ct., 1898

\section{PROVISIONAL SPECIFICATION.}

\section{Improvements in Apparatus employed in Wireless Telegraphy.}

We, Guglema Marconr, late of 67, Talbot Road, Westbourne Park, in the Countr of Middlesex, but now of 28, Mark Lane, in the City of London, Gentleman, and The Wireless Telegripir and Signal Co. Lo., of 28, Mark Laile, in the City of London, do hereby declare the nature of this invention to be as

5 follows:-

In the specification of a former patent granted to Guglielmo Marconi No. 12039 of 1896 is described an arrangement in which the transmitter consisted of a sparking appliance having one terminal connected to an insulated conductor in the air and the other terminal to earth, whilst the receiver contained a sensitive

10 tube or sensitive imperfect contact, having one end connected to a similar conductor and the other end to earth.

When both instruments are employed at the same station it is found that the sensitire tube or sensitive imperfect contact is liable to injury by its close proximitr to the sparking appliance.

15 In order to obriate this objection we enclose the receiver, containing the sensitive tube or sensitive imperfect contact, in a box of thick metal having only a small opening into it, and we employ the same conductor and earth plate for both instruments providing iswitches whereby these may be connected to either instrument at will, and we so arrange the apparatus that the switch lever in

20 the act of connecting the transmitter and disconnecting the receiver places a screen in front of the aperture.

Dated this 9 th day of December 1897.

G. MARCONI.

\section{COMPLETE SPECIFICATYON (AMENI)EI)).}

\section{Improvements in Apparatus employed in Wireless Telegraphy.}

We, Grguielzo Marconi, late of 67, Talbot Road, Westbourne Park, in the County of Middlesex, and now of 28, Nark Lane, in the City of London, Gentleman, and Tine Wireless Telegrapir and Srgiral Co., Li., of 28, Mark Lane, in the City of London, do hereby declare the nature of this invention and in

[Price 8d.] 
Improvements in Apparatus employed in Wireless Telegraphy.

what manner the same is to be performed to be particularly described and ascertained in and by the following statement:-

In the specification of a former patent granted to Guglielmo Marconi No. 12039 of 1896 is described an arrangement in which the transmitter consisted of a sparking appliance having one terminal connected to an insulated conductor in 5 the air and the other terminal to earth, whilst the receiver contained a sensitive tube or sensitive imperfect contact, having one end connected to a similar conductor and the other end to carth.

When both instruments are employed at the same station it is found that the sensitive tube or sensitive imperfect contact is liable to injury by its close proximity to the sparking appliance.

In order to obviate this objection we enclose the receiver, containing the sensitive tube or sensitive imperfect contact, in a box of thick metal having only a small opening into it, and we employ the same conductor and earth plate for both instruments providing iswitches whereby these mar be connected to 15 either instrument at will, and we so arrange the apparatus that the switch lever in the act of connecting the transmitter and disconnecting the receiver places a screen in front of the aperture.

Figure 1 is a plan (partly in section) of an apparatus arranged according to this invention and Figure 2 is a section on the line 2-2 Figure 1.

$a$ is a metal box preferably made of sheet iron about one sixteenth of an inch thick and $a^{1} a^{1}$ are apertures in it. In Figure 1 part of the top of the box is broken away to show part of the receiving instrument $b$ contained in it. $c$ is the sensitive tube of the receiver having its ends connected to insulated contacts $d d^{1}$. $e e$ are the balls of the sparking appliance or transmitter which are connected 25 to contacts $f f^{1}$ on insulating standards $f^{2} f^{3} . g$ is a switch lever of non-conducting material pivotted on the metallic top $g^{1}$ of an insulating standard $g^{2}$ and provided with a handle $h$ by which it is turned. $j j^{1}$ are contacts carried by the lever $g$ and connected to metallic plates $h^{1}$ which are in contact with the top $g^{1}$, the latter being connected to earth by the wire $l . m m^{1}$ are similar contacts carried by the lever $g$, and connected by the metallic plates $n$, and flexible wire $o$, to the insulated conductor or surface $p$, carried by the post $p^{1}$. When the instrument is to be used for transmitting as shown, the contacts $j$ and $m^{1}$ engage with the contacts $f f^{1}$, so that one of the balls $e$ is connected to earth, and the other to the conductor or surface $r$. Similarly when the instrument is to be used for receiving, the lever $g$ is turned about its pivot, and the contacts $j^{1}$ and $m^{1}$ enter the box through the apertures $a^{1} a^{1}$, and are made to engage with the contacts $d$ and $d^{1}$, so that the ends of the tube $c$ are connected one to earth and the other to the conductor or surface $p$.

If the apertures $a^{1} a^{1}$ are kept at a certain distance, say 15 inches from and out of the line with the sparking appliance, it will not be necessary to close them whilst transmitting, but this may readily be done when necessary by the arrangement shown in Figure 3 where $q q$ are shutters pivotted to the box at $q^{1} q^{1}$ and operated by a cord $r$ led over pulleys and connected to the lever $g$.

If a telegraphic instrument outside the box be employed the connections to it from the receiver should be made by a third pair of contacts on the lever as no wires should be allowed to protrude from the box.

The above described arrangement can be simplified by connecting one terminal of the receiver permanently to the box. The corresponding aperture of the box can then be permanently closed, and the contact $j^{1}$ or $m^{1}$ of the switch instead of passing through the aperture is caused to clamp on to a piece of metal fixed on the outside of the box. Or the box may be permanently connected to earth or to the insulated surface or conductor. In this case one pair of contacts on the lever can be dispensed with, if one of the balls of the oscillator is also permanently connected to earth or the insulated surface or conductor.

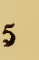


Improvements in Apparatus employed in Wireless Telegraphy.

receiver and transmitter have to be connected to a surface or surfaces (whether insulated or not) or to the earth.

Haring now particularly described and ascertained the nature of our said invention and in what manner the same is to be performed, we are aware that it 5 has previously been proposed, in a system of wireless telegraphy, (1) to protect the receiver from the effect of the local transmitter by enclosing it in a metallic box, (2) to use the same conducting surface or surfaces for both instruments, (3) to connect and disconnect the two instruments by means of a change-over switch, and (4) to open and close apertures in the metallic box containing the

10 receiver by means operated by the switch lever, and we wish it to be understood that we make no general claim to such arrangement or combination of apparatus but we declare that what we claim is:-

1. In apparatus comprising a transmitter, a receiver, a metallic box containing the receiver, a conducting surface, and a switch, providing an automatic shutter

15 which closes the box when the transmitter is being used.

2. Apparatus substantially as described and illustrated in the drawings.

Dated this 9th day of September 1898.

G. MARCONI.

Redhill : Printed for Her Majesty's Stationery Office, by Malcomson \& Co., Ltd.-1899 






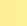





